IFT-2000-22

hep-ph/0009201

\title{
THE LIGHT HIGGS WINDOW IN THE 2HDM AT GigaZ
}

\author{
MARIA KRAWCZYK \\ Institute of Theoretical Physics, University of Warsaw, \\ Warsaw, 00-681, Poland \\ PETER MÄTTIG \\ Weizmann Institute, Rehovot, Israel \\ and \\ JAN ŻOCHOWSKI \\ Faculty of Physics, Biatystok University, \\ Biatystok, Poland
}

\begin{abstract}
The sensitivity to a light Higgs boson in the general 2HDM (II), with a mass below $40 \mathrm{GeV}$, is estimated for an future $e^{+} e^{-}$linear collider operating with very high luminosity at the $Z$ peak (GigaZ). We consider a possible Higgs boson production via the Bjorken process, the $(h A)$ pair production, the Yukawa process $Z \rightarrow b \bar{b} h(A), \rightarrow \tau \bar{\tau} h(A)$, and the decay $Z \rightarrow h(A)+\gamma$. Although the discovery potential is considerably extended compared to the current sensitivities, mainly from LEP, the existence of a $h$ or $A$ even with a mass of a few GeV cannot be excluded with two billion $Z$ decays. The need to study the very light Higgs scenario at a linear $e^{+} e^{-}$collider running at several hundred $\mathrm{GeV}$ and the LHC is emphasised.
\end{abstract}

\section{Introduction}

Whereas for the minimal version of the Standard Model with just one scalar (Higgs) doublet, data require the Higgs boson mass to be above about $113 \mathrm{GeV}$ [1], no or less stringent limits can be set for more complicated sectors. A fairly straight-forward extension of the Higgs sector in the Standard Model is, for example, to assume two instead of one scalar doublets. For a CP conserving model this implies five physical bosons, two neutral scalars $h$ and $H$ (with $M_{h}<M_{H}$ ), one neutral pseudoscalar $A$, and two charged Higgs bosons $H^{ \pm}$. Apart from the masses of these bosons, the model is unambiguously defined by specifying $\tan \beta$, given by the ratio of the vacuum expectation values of the two doublets, the angle $\alpha$ describing the mixing in the neutral scalars sector, and one Higgs boson self-coupling, say $g_{h H^{+} H^{-}}$[2].

In the context of the minimal supersymmetric model (MSSM) a similar Higgs sector with five physical Higgs bosons exists. Relations between the parameters, including the 
Higgs masses, are induced from the structure of the superpotential. These relations reduce the number of independent parameters at tree level to just two. As a result, for some benchmark parameter sets of the MSSM, e.g. maximal stop mixing scenario and $M_{S U S Y}$ $=1 \mathrm{TeV}, m_{\text {top }}=175 \mathrm{GeV}, M_{h}$ and $M_{A}$ are constrained by data to be larger than about 90 $\mathrm{GeV}$ and $\tan \beta$ to lie between about 0.5 and 2.3 [1]. The phenomenological consequences of the present measurements are quite different in the general 2HDM (Model II). An analysis in this framework of the current constraints from LEP1 data, and from other experiments can be found in 3, [4. In particular, present LEP data are unable to rule out that either $h$ is light but $M_{A} \gtrsim M_{Z}$ or vice versa. Additional constraints for a very light $h$ or $A$, arise from low energy experiments like $g-2$ of muons or searches for $\Upsilon$ decays into $M_{h}$ and $M_{A}$, see [5, 6]. Experiments at other than LEP colliders have hardly any sensitivity to a light 2HDM Higgs boson [7]. Even after combining all existing experimental information there is still a large range in the parameter space to which no experimental sensitivity exists and a window is left in the 2HDM(II) model for a light Higgs boson extending down to even massless $h$ or $A$.

Here we estimate the prospects for exploring the parameter space of 2 HDM (II) at the proposed TESLA I linear $e^{+} e^{-}$collider [9] running at the $Z$ peak, deemed GigaZ, with a luminosity of about two orders of magnitude higher than LEP [10]. In addition to the higher luminosity improved charm and bottom tagging capability will push the experimental sensitivity to considerably smaller cross sections for the Higgs production processes. We will study the potential for finding a light Higgs boson in particular for the process $Z \rightarrow h(A)+\gamma$, sensitive to both large and small tan $\beta$ extrapolating our analysis of LEP data [3], but also discuss other important Higgs production processes at the $Z$. At this stage no detailed simulation studies of future measurements are performed but the sensitivities are estimated by extrapolating existing LEP measurements. In addition we will briefly comment on the potential signatures at the Linear Collider operating at high energies of several hundred $\mathrm{GeV}$ and on implications of the very light Higgs boson scenario at LHC.

\section{Some assumptions on experimentation at GigaZ}

We assume TESLA running at the $Z$ mass with an instantanous luminosity of $7 \cdot 10^{33}$ $\mathrm{sec}^{-1} \mathrm{~cm}^{-2}$ 10 . For a nominal year of 100 days this implies some two billion $Z$ 's to be produced, about a factor 500 more than what has been collected by each LEP experiment during five years of operation. The forseen performance of a TESLA detector is basically described in [9].

Pertinent for this study is the potential for tagging bottom and charm quarks, which has been estimated in [11, and the photon energy resolution. The possible efficiencies are given as a function of the remaining background from the other flavours. Without attempting to optimise the working point of the tagging algorithm, we assume the following performance. The bottom tagging efficiency of $60 \%$ implies that only $2 \%$ of charm

${ }^{1}$ Although we will refer in this paper to the specific TESLA scheme, our arguments apply equally well to the other proposals for a Linear Collider [8]. 
quarks and $0.2 \%$ of light quarks will be retained. Charm tagging has only been used with marginal efficiencies and purities at LEP. However, the SLC experiment has shown the virtues of a Linear Collider also for charm tagging. Experiments at TESLA are expected to tag charm with an efficiency of $50 \%$ while accepting only $15 \%$ of bottom and $0.8 \%$ of light quarks. No improvement is expected for tau identification. Isolated photons should be easily identifiable and we assume an energy resolution of $d E / E=0.1 / \sqrt{E}$ [9], typically a factor $1.5-2$ better than at LEP experiments.

We do not explicitely consider additional background sources. However, beamstrahlung and underlying two-photon interactions may become important in view of the very small cross sections to be considered.

\section{Higgs production processes in $Z$ decays}

There are potentially four relevant mechanisms for the lightest Higgs boson production at the Linear Collider running at the $Z$-peak. They cover complementary regions in the $2 \mathrm{HDM}$ parameter space. The Higgs strahlung $Z \rightarrow Z h$ has basically the same experimental features as the Standard Model Higgs boson production. In addition there is the pair production $Z \rightarrow A h$ important for $M_{A}+M_{h} \leq M_{Z}$. Both of these are considered as the main production processes in the framework of the MSSM. The production yields of these processes are proportional to $\sin ^{2}(\beta-\alpha)$ and $\cos ^{2}(\alpha-\beta)$, respectively.

Both $A$ and $h$ can be singly produced in two other processes. Since no relation between $M_{h}$ and $M_{A}$ exists, those processes are of particular interest within the 2HDM. For $\tan \beta>1$ and low mass Higgs bosons, the Yukawa processes $Z \rightarrow \tau^{+} \tau^{-} h(A), b \bar{b} h(A)$, are promising. These processes depend on both $\sin (\beta-\alpha)$ and $\tan \beta$ for $h$ t, and for $A$ on just $\tan \beta(1 / \tan \beta)$ for down (up) type fermions [12]. The radiative $Z$ decays $Z \rightarrow h(A)+\gamma$ is sensitive to both $\sin (\beta-\alpha)$ and values of $\tan \beta$, see [3].

Extrapolating from existing LEP studies we will derive constraints on the parameters of the 2HDM for masses between 5 and $40 \mathrm{GeV}$. We will always refer to the $95 \%$ confidence exclusion limit. Note that the potential discovery of, say, a five standard deviation Higgs signal excess over the Standard Model background is only possible in a parameter space which is smaller than the one for exclusions.

\subsection{Direct $Z$ decays into Higgs bosons}

The Higgsstrahlungs process

$$
Z \rightarrow Z h
$$

is the main channel to search for a Standard Model Higgs boson at LEP. In the 2HDM the yield is suppressed by $\sin ^{2}(\alpha-\beta)$ compared to the Standard Model rate. Current

\footnotetext{
${ }^{2}$ The corresponding coupling is proportional to $-\sin \alpha / \cos \beta=\sin (\beta-\alpha)-\tan \beta \cos (\beta-\alpha)$ for down type fermions. For up type fermions: $-\tan \beta \rightarrow+1 / \tan \beta$.
} 
LEP data [13] yield minimum values of $\sin ^{2}(\alpha-\beta) \sim \mathcal{O}(0.006-0.01)$ for $5 \leq M_{h} \leq 20$ $\mathrm{GeV}$ and somewhat worse $\sim \mathcal{O}(0.006-0.06)$ for $20<M_{h}<40 \mathrm{GeV}$. For the lowest masses $M_{h}<5 \mathrm{GeV}$ direct searches yield somewhat less restrictive limits of about 0.01 , however, more restrictive limits of $\sim 5 \cdot 10^{-3}$ are derived from the $Z$ line shape [14]. The cleanest way to detect the Higgsstrahlungs process for not too low Higgs masses is by tagging $Z$ decays with electron or muon pairs, each of which should yield at GigaZ some $6000 \cdot \sin ^{2}(\beta-\alpha)$ events of the type $e^{+} e^{-} h, \mu^{+} \mu^{-} h$ each for $M_{h} \sim 5 \mathrm{GeV}$. The signature would then be two highly energetic leptons with a mass close to $M_{Z}$ and some hadrons, respectively two $\tau$ leptons. Background is due to initial and final state (off shell) photon radiation with the photon decaying into a pair of fermions. In addition one has to consider the potential overlap of an annihilation event with an event from two photon interaction. Other decays of the $Z$ can also be tagged with additional experimental effort, in particular those into neutrinos and taus provide rather clean signals. However, trigger efficiencies and backgrounds have to be studied in more detail. If hadronic decays of the $Z$ are included in the search, jets from gluon radiation are the most important and rather uncertain background.

Compared to LEP the sensitivity at the TESLA Linear Collider should improve by $\sqrt{\mathcal{L}_{L C} / \mathcal{L}_{L E P}} \sim 20$ for a light Higgs boson and additional factors if the Higgs boson decays into charm and bottom quarks. For $M_{h} \geq 10 \mathrm{GeV}$ and $\tan \beta>1$, the $h$ decay into bottom is preferred. For $M_{h} \geq 5 \mathrm{GeV}$ and $\tan \beta<1, h$ decays mostly into charm quarks. Extrapolationg from current LEP limits we estimate that at TESLA the range of $s_{L C}^{2}=\sin ^{2}(\alpha-\beta)>5 \cdot 10^{-4}$ could be covered for all masses below $M_{h} \sim 10 \mathrm{GeV}$. For higher masses the limits should become less stringent, reaching $\sim 0.005$ for $M_{h} \sim 40$ $\mathrm{GeV}$. This limit is almost independent of $\tan \beta$. In the following discussion we assume $s_{L C}^{2}\left(M_{h}\right)=\sin ^{2}(\alpha-\beta)_{L E P}\left(M_{h}\right) / 20$, where $\sin ^{2}(\alpha-\beta)_{L E P}$ is the limit from LEP [13]. If a light $h$ exists and is not found in the Higgsstrahlungs process it implies that $h$ almost decouples from the $Z$.

If instead $M_{h}$ is large and the pseudoscalar $A$ is light, the search for $Z \rightarrow Z h$ does not constrain $\sin ^{2}(\alpha-\beta)$. Since no $Z Z A$ coupling exists, direct $A$ production in $Z$ decays can only occur via

$$
Z \rightarrow A h
$$

whose yield is proportional to $\cos ^{2}(\alpha-\beta)$. If such a decay is kinematically possible, no significant improvement over LEP limits can be expected from GigaZ. If $M_{h}>M_{Z}$ the mass of $A$ is unconstrained.

\subsection{The Yukawa Process}

The pseudoscalar $A$ can be singly produced by radiation off heavy fermions. The same is true for $h$ leading to complementary constraints compared to the Higgsstrahlungs process. Since the production yield is proportional to the Yukawa coupling $f \bar{f}(h, A)$ we will refer to this process as Yukawa process. The experimentally most prominent decays are

$$
Z \rightarrow b \bar{b} h(A), \quad \tau^{+} \tau^{-} h(A) .
$$


For $\sin ^{2}(\alpha-\beta)=0$ the cross sections scale with $\tan ^{2} \beta$. At LEP preliminary analyses have been presented for $A$ by ALEPH [15] and for both $A$ and $h$ by DELPHI [16].

The dominant search channels are

$$
\begin{array}{ccc}
Z \rightarrow b \bar{b}^{-} \tau^{+} \tau, \tau^{+} \tau^{-} \tau^{+} \tau^{-} & \text {for } M_{h} \leq 10 \mathrm{GeV} \\
Z \rightarrow & b \bar{b} b \bar{b} & \text { for } M_{h} \geq 10 \mathrm{GeV}
\end{array}
$$

The main Standard Model background will be $Z$ decays into quarks, particularly bottom pairs with the additional emission of one or two hard gluons. For very low Higgs masses also the background from overlapping $Z$ decays and two - photon interactions may become important. Since the mass resolution of the $\tau^{+} \tau^{-}$and $b \bar{b}$ systems from a potential $h$ or $A$ decay is marginal, the signal has essentially to be derived from an excess of the inclusive event yield of the candidate topology. At the Linear Collider with its higher luminosity and higher bottom tagging efficiency such a search may finally be limited by the knowledge of the irreducible background processes.

For masses $M_{h, A}<10 \mathrm{GeV}$ and $\tan \beta>1$, the Higgs bosons will mainly decay into a pair of $\tau$ leptons. Current analyses at LEP include hadronic $\tau$ decays. To separate the Higgs signal from background one has to understand yield and kinematic features of low multiplicity gluon jets. The uncertainty in their yield may be a limiting factor. For masses larger than $10 \mathrm{GeV}$ and $\tan \beta>1$, the Higgs boson will predominantly decay into a pair of bottom quarks. In this case the main background will be due to $b \bar{b} g$ with the gluon splitting into $b \bar{b}$. Its total cross section is $\sim 10 \mathrm{pb}$, which has to be compared with a cross section of $\sim 0.01 \cdot \tan ^{2} \beta$ pb for $M_{h, A} \sim 10 \mathrm{GeV}$, decreasing with the Higgs mass. The background may be suppressed by selecting special kinematical event properties. However, one has to be aware that, apart from uncertainties of $10-30 \%$ in the overall cross section for gluon splitting, there are also uncertainties as to how gluons hadronise into bottom particles.

An extrapolation to GigaZ is somewhat uncertain because of future theoretical developments and experimental ideas to measure the yield and properties of $g \rightarrow b \bar{b}$. From the current understanding we estimate that at GigaZ limits of $\tan \beta>\mathcal{O}(5)$ can be obtained for Higgs masses between 5 and $10 \mathrm{GeV}$. Above the bottom threshold it will be difficult to reach sensitivities below $\tan \beta=10$ for $M_{h, A} \sim 10 \mathrm{GeV}$, respectively $\tan \beta=30$ for $M_{h, A} \sim 40 \mathrm{GeV}$. The corresponding limits are included in Figs.1‥13.

No LEP analysis exists on the Yukawa process for $\tan \beta<1$. In this range $h$ and $A$ would decay predominantly into charm quarks since its coupling is proportional to $1 / \tan \beta$. Charm tagging at LEP is substantially less efficient than bottom tagging. But even for the improved charm tagging at the Linear Collider any sensitivity to such a decay will be extremely difficult because of the significant irreducible background $Z \rightarrow c \bar{c} c \bar{c}$.

\section{$3.3 \quad Z \rightarrow h(A)+\gamma$}

This radiative decay of $Z$ proceeds for $h$ via loops of $\mathrm{W}$ - bosons and fermions. In the Standard Model with one doublet the W - loop is by far the dominant one, in the 2HDM 
it is suppressed by $\sin ^{2}(\beta-\alpha)$. The $\mathrm{W}$ - loop interfers negatively with the fermion loops in $\mathrm{SM}$, in $2 \mathrm{HDM}$ with small $\sin (\beta-\alpha)$ destructive interference occurs only with up-type quarks (obviously with the largest effect for the top). The fermion contributions may be enhanced relative to the Standard Model by $\sim \tan ^{2} \beta$ (down type fermions) or $\sim 1 / \tan ^{2} \beta$ (up type fermions, particularly top quark) 3 . For the radiative $\mathrm{Z}$ decay into the pseudoscalar $A$ only the fermion loops contribute. In the 2HDM additional contributions are due to loops of charged Higgs bosons for $h$ [3]. These are of minor importance for a heavy $M_{H}^{ \pm}$above $300 \mathrm{GeV}$ as derived from the $b \rightarrow s \gamma$ yield [21], and a light Higgs boson $h$.

All LEP experiments have searched for radiative $Z$ decays [17 20]. Their sensitivities are determined by the potential to observe a narrow resonance over a smoothly varying, irreducible background mostly due to photons emitted from the final state fermions in standard $Z \rightarrow f \bar{f}$ decays. Therefore, for all kinds of fermions, the luminosity and the mass resolution of the $f \bar{f}$ system determine the quality of the measurement. The envisaged luminosity at GigaZ, being two orders of magnitude higher than the one at LEP, will push the reach to this process significantly beyond existing limits. An additional asset of the LC, depending on the decay mode of the Higgs boson, is the increased capability of identifying fermion types.

The mass of the $f \bar{f}$ system, i.e. potentially the Higgs mass, given by $M_{f \bar{f}}=$ $E_{c m} \sqrt{1-2 E_{\gamma} / E_{c m}}$, is best determined, at least for masses larger than about $30 \mathrm{GeV}$, through a precise measurement of the photon energy $E_{\gamma}$. For smaller masses the best resolution is obtained by a kinematic fit to the three body system of fermions and the photon. Here presumably no improved resolution will be possible compared to current LEP measurements. As discussed above, because of the smaller beam pipe diameter and more sophisticated micro vertex detectors, the heavy quark tagging capability at the Linear Collider is largely enhanced compared to LEP. This will allow one to reduce the background significantly if the Higgs decays into bottom or charm.

Assuming no signal will be observed, the limits $S_{L C}$ to be set at a Linear Collider improve compared to those at LEP, $S_{L E P}$, according to

$$
S_{L C}=S_{L E P} \sqrt{\frac{\mathcal{L}_{L E P}}{\mathcal{L}_{L C}}} \sqrt{\frac{\left(d M_{f \bar{f}}\right)_{L C}}{\left(d M_{f \bar{f}}\right)_{L E P}}} \frac{\epsilon_{\text {signal }}^{L E P}}{\epsilon_{\text {signal }}^{L C}} \frac{\sqrt{\epsilon_{\mathrm{bck}}^{L C}}}{\sqrt{\epsilon_{\mathrm{bck}}^{L E P}}}
$$

where $\mathcal{L}$ denotes the integrated luminosities at the respective collider, $d M_{f \bar{f}}$ the mass resolution of the $f \bar{f}$ system, and $\epsilon_{\text {signal }}$ and $\epsilon_{\mathrm{bck}}$ the corresponding efficiencies for signal and background. Thus, depending on the decay mode, one year of LC running will boost the sensitivity by factors of about 30 for $(q \bar{q})_{\text {inclusive }}$, i.e for all decays into quarks or gluons, and for decays into $\tau$ pairs, 36 for those into charmed quarks and 50 for those into $b \bar{b}$ pairs.

The $95 \%$ confidence limits for the product branching ratio $\operatorname{Br}(Z \rightarrow(h, A)+\gamma) \operatorname{Br}((h, A) \rightarrow$ $\bar{f} f$ ) can fairly well be described by a function of the form

$$
S_{M_{h, A}}=K \cdot \exp \left[B \cdot\left(M_{h, A}-30 G e V\right)\right],
$$

\footnotetext{
${ }^{3}$ Strictly speaking this proportionality holds only for small $\sin (\beta-\alpha)$.
} 
with $K, B$ constants depending on the Higgs decay mode and $M_{h, A}$ in GeV. The expected sensitivities are shown in Fig. 1. They will be used below to constrain the scalar $h$ and pseudoscalar $A$ production. As a figure of merit also the rate in the Standard Model with just one Higgs boson is shown. In principle a Standard Model Higgs boson is in reach through this process, but, of course, for the accessible mass range it is already excluded by LEP1 data.

\subsection{Constraints of the 2HDM parameters at GigaZ}

The expected sensitivity ranges at GigaZ for $h, A$ are depicted in Figs. 땅.

In Fig. 1 the potential exclusion range for a scalar Higgs of $M_{h}=6,12$, or $20 \mathrm{GeV}$ is shown in the $\left(\tan \beta, \sin ^{2}(\beta-\alpha)\right)$ plane. These figures exhibit the complementarity of the considered processes. The Higgsstrahlung $Z \rightarrow Z h$ bounds the value of $\sin ^{2}(\beta-\alpha)$, excluding the region indicated at the right of the figures. For the remaining allowed region of very small $\sin ^{2}(\beta-\alpha)$, the Yukawa process sets a lower limit on $\tan \beta$ excluding the upper part of the figures. The radiative process $Z \rightarrow h+\gamma$ is sensitive to both $\tan \beta$ and $\sin ^{2}(\beta-\alpha)$. Its exclusion range is unique for $\tan \beta<1$ and $\sin ^{2}(\beta-\alpha) \rightarrow 0$. It should be noted that if a light $h$ is found at GigaZ the complementarity of these processes allows one to significantly constrain the parameters in the 2HDM. Also shown in Fig. 1 are the current constraints from LEP, as discussed in previous sections. Obviously the GigaZ can significantly extend the excluded region of the parameter space.

The potential exclusion region for $h$ in the $\left(\tan \beta, M_{h}\right)$ plane is shown in Fig. 2. Here constraints from $Z \rightarrow h+\gamma$ were obtained under the most conservative assumptions of $\sin (\beta-\alpha)=0$ and a mass of the charged Higgs boson of $300 \mathrm{GeV}$. In the range $\tan \beta>1$ the most constraining result comes from the Yukawa process, however does not reach below $\tan \beta \sim 5$ for $M_{h}<10 \mathrm{GeV}$ and $\tan \beta=10-30$ for higher masses. For $\tan \beta<1$ limits are due to the radiative $\mathrm{Z}$ decay and are of $\mathcal{O}(0.2-0.1)$, rather independent of $M_{h}$. The sensitivity of the $Z \rightarrow h+\gamma$ decay for $\tan \beta>1$ is indicated by the dashed line and is seen to be less constraining than the Yukawa process. Also shown is the current limit from LEP revealing a substantial gain at GigaZ. However, if the $h$ decouples from the $Z$, i.e. $\sin ^{2}(\beta-\alpha) \sim 0$ a sizeable parameter space for the scalar Higgs $h$ remains uncovered a light neutral Higgs boson in the 2HDM remains a possibility.

A similar picture emerges for the pseudoscalar $A$ shown in Fig. 3. The main difference is that its sensitivity range is independent of $\sin ^{2}(\beta-\alpha)$. The Yukawa process yields similar exclusion potential for $h$ and $A$ in the region $\tan \beta>1$. Also in this case the radiative decay has a smaller reach than the Yukawa process. The sensitivity to $A$ is larger than the one for $h$ because of the absence of the negative interference of top and $W$ loops. Out of the same reason also the exclusion in the $\tan \beta<1$ region is somewhat better $\mathcal{O}(0.3-0.2)$. Combining all constraints, also a light $A$ cannot be excluded for all 2HDM parameters at GigaZ. As in the case for $h$, GigaZ improves significantly over LEP, whose limits are indicated by the full line. 


\section{The High Energy frontier}

Up to now we have only considered the potential of finding a light Higgs boson $h$ or $A$ at the $Z$ peak. Although the sensitivity to a light $h$ or $A$ can be significantly extended at GigaZ, even two billion produced $Z$ 's are not enough to definitely find or rule out a very light $h$ or $A$ in the framework of the $2 \mathrm{HDM}$ (II). On the other hand, if such a light Higgs boson exists but is not found, the sensitivity reach at GigaZ would imply $\mathcal{O}(0.2) \leq \tan \beta \leq \mathcal{O}(20)$. If the light Higgs boson is a scalar $h$, then in addition $\sin ^{2}(\alpha-\beta) \sim 0$, i.e. the $h$ decouples from the $Z$.

Apart from the GigaZ option other future projects are under consideration. Potentials for the discovery of a light Higgs boson in 2HDM (II) in $\gamma \gamma$, and $\mu^{+} \mu^{-}$colliders are discussed in [22]. Improved constraints may also follow from low energy high precision experiments, e.g. the E821 experiment for $g-2$ for muon at BNL [23. All those experiments can increase the sensitivity, but they cannot cover the whole parameter space of the 2HDM for a light Higgs boson. Thus, if one does not observe the Higgs boson, one will not be able to rule out the light Higgs scenario. Here we want to briefly comment on possibilities for exploring the $2 \mathrm{HDM}$ with the higher energies available both at TESLA and the LHC. In most cases a very light Higgs scenario, discussed in this paper, has been neglected in prospective studies for these machines. More detailed considerations are needed for firm conclusions on the potential for probing the very light Higgs scenario. We will suggest some lines of studies in the following paragraphs.

At a high energy $e^{+} e^{-}$collider the direct production of a light $h$ or $A$ via the pair production $h A$ with either $A$ or $h$ being light and the other Higgs boson from the pair having a mass of a few hundred $\mathrm{GeV}$, and the $t \bar{t}(h, A)$ Yukawa process may probe new regions of the $2 \mathrm{HDM}$ parameter space. In the first reaction the final decay products can be, for example, $W^{+} W^{-}(A, h), Z Z(A, h)$. The second process exploits the strong Yukawa coupling to top quarks. The emission of a $h$ or $A$ from top quarks can be searched for with the Higgs boson decaying into pairs of bottom, charm or tau. Here again one has to discriminate against background from gluon splitting into quarks. A related signal is a possible enhancement at the $t \bar{t}$ threshold due to virtual Higgs boson exchange 26]. To understand the precision and reach of such processes, more detailed studies including experimental effects and updated parameters have to be performed 1 .

There are plenty of channels at $p p$ and $p \bar{p}$ colliders in which a light Higgs boson may be produced with a sizeable event yield. However, it is not obvious if those can be isolated from the formidable background. Most studies have been performed for $M_{h, A}>100 \mathrm{GeV}$ and indicate that it is increasingly difficult to detect a Higgs signal the smaller the Higgs mass is. On the other hand detailed studies for masses of a few $\mathrm{GeV}$ are missing. The only analysis so far at $p p$ colliders with relevant limits has recently performed by the CDF collaboration and yields limits within the MSSM from the Yukawa process $b \bar{b} h$ for large $\tan \beta$ and for Higgs masses larger than $70 \mathrm{GeV}$ 28].

\footnotetext{
${ }^{4}$ Within a $\mathrm{CP}$ non conserving $2 \mathrm{HDM}$ the sensitivity at a $500-800 \mathrm{GeV}$ Linear Collider has been theoretically considered in 227. Also their study, not particularly focused on the very light Higgs scenario, indicates that from the Bjorken process, pair production and the Yukawa process the whole parameter space cannot be covered.
} 
As discussed in [4] a global fit to current precision EW measurements performed in the $2 \mathrm{HDM}(\mathrm{II})$ allows the existence of a very light $h$ or $A$, with mass even below $20 \mathrm{GeV}$, while the other Higgs bosons may have masses of several hundred GeV. The detection of such massive Higgs bosons may be possible at high energy colliders as TESLA or the LHC. These heavy Higgs bosons may reveal the first signal of the 2HDM sector and open up the window for the exploration of a very light Higgs boson, discussed in this paper.

Analyses in the framework of the MSSM have shown a good sensitivity both at TESLA and LHC to such massive bosons if they decay directly into gauge bosons or $b \bar{b}$ pairs [24, 25]. These studies also basically apply to the 2HDM. The existence of a light Higgs boson in the general $2 \mathrm{HDM}$, however, opens up the possibility of cascade decays which result into more involved decay patterns. Decays like $H \rightarrow h h, A \rightarrow Z h, H^{ \pm} \rightarrow W^{ \pm} h$ (for a light $h$ ), respectively $h \rightarrow A A, h \rightarrow Z A$ or $H^{ \pm} \rightarrow W^{ \pm} A$ (for a light $A$ ) are more complicated to reconstruct. The feasibility of identifying these decays requires additional studies. Those performed in the LHC framework, again at this stage only in the MSSM, indicate that it may be difficult to cover the whole parameter space. At TESLA these events could have quite distinct features and be relatively simply to identify.

In conclusion we want to reemphasise that the case of a very light Higgs boson, i.e. with a mass of $40 \mathrm{GeV}$ or below - even only a few $\mathrm{GeV}$, is not closed. A $Z$ factory like GigaZ producing two billion $Z$ 's per year will allow one test a significantly larger parameter space of the $2 \mathrm{HDM}$ compared to what has yet been probed. However, it seems unlikely that the whole space can be covered. A window for a very light Higgs boson remains. It may be possible to study the whole range with the high energies provided at TESLA and LHC, however, most studies so far have not considered very light Higgs bosons. More definite conclusions can only be reached if detailed analyses are performed taking into account the light mass Higgs window.

\section{Acknowledgements}

We thank Piotr Chankowski for important and enlightening discussions on parameters of 2HDM. MK thanks Michael Kobel for informing of and sending the paper [5]. We are grateful to J. Kühn and M. Jeżabek to make us aware of the sensitivity of the $t \bar{t}$ threshold to the light Higgs. MK would like to thank Padova University for warm hospitality during the preparation of this paper. This work was partly supported by Polish Committee for Scentific research, grant No 2P03B01414.

\section{References}

[1] The ALEPH, DELPHI, L3 an OPAL Collaborations, Searches for the Higgs Boson: Preliminary combined results using LEP data collected at energies up to $209 \mathrm{GeV}$, submitted to ICHEP'2000, Osaka, Japan, July 27 - August 2,2000, presented by K.Hofmann and P.Igo-Kemenes. 
[2] J. F. Gunion, H. E. Haber, G. Kane and S. Dawson, "The Higgs Hunter's guide" Addison-Wesley (1990);

A. Djouadi, V. Driesen, W. Hollik and A. Kraft, Eur. Phys. J C1 (1998) 163

[3] M. Krawczyk, J. Żochowski, P. Mättig, Eur. Phys. J C8 (1999) 495 (hep-ph/9811256).

[4] P. H. Chankowski, M. Krawczyk, J. Żochowski, Eur. Phys. J C11 (1999) 661 hepph/9905436).

[5] S. M. Keh, Tau physics with the Crystal Ball Detector, DESY F31-86-6.

[6] M. Krawczyk, J. Żochowski, Phys. Rev. D55 (1997) 6968 (hep-ph/9608321).

[7] A. C. Bawa, M. Krawczyk, Phys. Lett. B357 (1995) 637

[8] S.Kuhlman et al., hep-ex/9605011

[9] Conceptual Design Report of a $500 \mathrm{GeV}$ e+e- Linear Collider with Integrated Xray Laser Facility, DESY 1997-048, ECFA 1997-182; Editors: R. Brinkmann, G. Materlik, J. Rossbach, A. Wagner.

[10] N.Walker, Talk at the ECFA/DESY Linear Collider Workshop, Frascati, 8-10 November 1998;

R.Brinkmann, Talk at the ECFA/DESY Linear Collider Workshop, Orsay 1999.

[11] R.Hawkings, Vertex detector and flavour tagging studies for the TESLA linear collider, LC-PHSM-2000-021-TESLA.

[12] H.E.Haber, in Perspectives on Higgs Physics II, Gordon L. Kane ed., Singapore, 1997, hep-ph/9707213.

[13] The L3 Collaboration, M. Acciarri et al., Phys. Lett. B385 (1996) 454.

[14] The OPAL Collaboration, G.Abbiendi et al., Two Higgs Doublet Model and Model Independent Interpretation of Neutral Higgs Boson Searches, CERN-EP-2000-092.

[15] The ALEPH Collaboration, Search for a Light Higgs Boson in the Yukawa Process, PA13-027, Contribution to the International Conference on High Energy Physics, Warsaw, Poland, 25-31 July 1996.

[16] The DELPHI Collaboration, Search for Yukawa production of a light neutral Higgs at LEP1, DELPHI 99-76 CONF 263, Paper submitted to the HEP'99 Conference, Tampere, Finland, July 15-21.

[17] The ALEPH Collaboration, R.Barate et al., Eur. Phys. J C4 (1998) 571.

[18] The DELPHI Collaboration, J.A.Barrio et al., internal note DELPHI 95-73 PHYS 508, submitted to the EPS-HEP conference '95.

[19] The L3 Collaboration, M.Acciarri et al., Phys. Lett. B388 (1996) 409.

[20] The OPAL Collaboration, G.Alexander et al., Z. Phys. C71 (1997) 1. 
[21] The CLEO Collaboration, S. Alam, Phys. Rev. Lett.74 (1995) 2885;

The CLEO Collaboration, CONF 98-17, ICHEP98 1011, submitted to the ICHEP conference Vancouver, 1998;

The ALEPH Collaboration, R.Barate et al., Phys. Lett. B429 (1998) 169;

M. Misiak, S. Pokorski and J. Rosiek, Heavy Flavors II, eds. A.J. Buras and M. Lindner, p. 795 (hep-ph/9703442);

M. Ciuchini et al., Nucl. Phys. B527 (1998), 21.

[22] D.Choudhury and M.Krawczyk, Phys. Rev. D55 (1997)2774;

I.F. Ginzburg, M.Krawczyk, P.Osland, Resolving SM-like scenarios via Higgs boson production at Photon Colliders: I. 2HDM vs SM, IFT-2000-21;

M.Krawczyk, Testing 2HDM at Muon Colliders, on proc. Workshop on Physics at the First Muon Collider and at the Front End of the Muon Collider, Batavia, IL, 6-9 Nov 1997, p. 635 (hep-ph/9803484).

[23] R. Carey, talk at ICHEP 2000, Osaka, Japan, July 27 - August 2,2000.

[24] E.Accomando et al., Phys.Rept. 299 (1998) 1.

[25] E.Richter-Was et al. I.J.M.P. A13 (1998) 1371;

ATLAS-Collaboration Detector and Physics Performance Technical Design Report, CERN/LHCC/99-15, 25 May 1999;

R.Kinnune and D.Denegri, CMS Note 1997/057.

[26] M.Jeżabek and J.H.Kühn, Phys. Lett. B316 (1993) 360;

M.J.Strassler and M.E.Peskin, Phys. Rev. D43 (1991), 1500;

and references therein.

[27] B.Grzadkowski, J.Gunion, J.Kalinowski, Phys. Lett. B480 (2000) 287; Phys. Rev. D60 (1999) 075011.

[28] J.Valls, The CDF Collaboration, FERMILAB-CONF-00/106-E;

Published Proceedings 35th Rencontres de Moriond: Electroweak Interactions and Unified Theories, Les Arcs, France, March 11-18, 2000. 

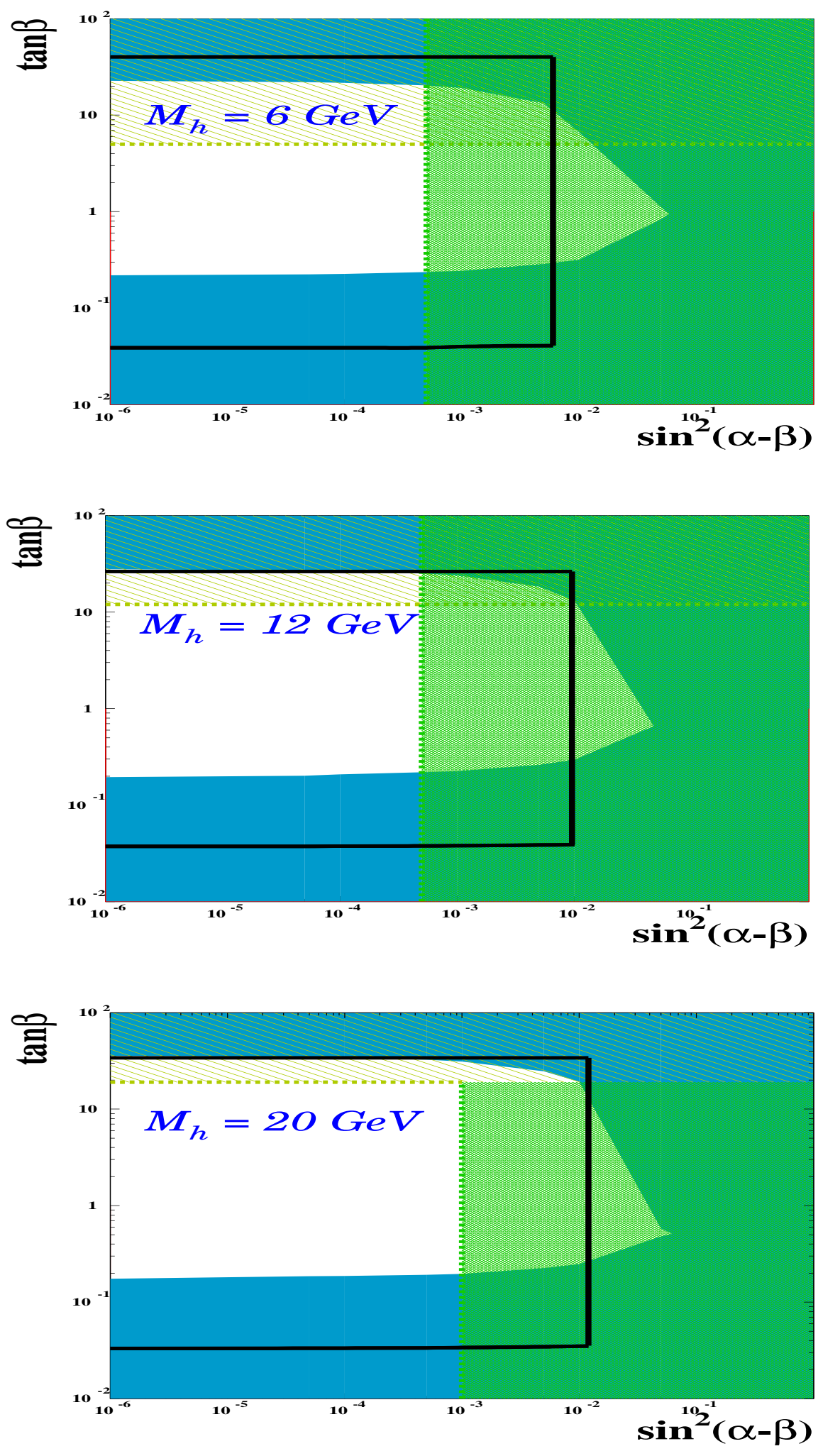

Figure 1: Limits on $\sin ^{2}(\beta-\alpha)$ and $\tan \beta$ for a light scalar $h$ of mass $M_{h}=6,12$, and 20 $\mathrm{GeV}$ at GigaZ. The light hatched (yellow) region shows the exclusion from the Yukawa process, the cross hatched (green) region the one from Higgsstrahlung, the dark area depicts the exclusion range from $Z \rightarrow \gamma h$ (with mass of $H^{ \pm}=300 \mathrm{GeV}$ ). Also shown are the current best limits from LEP (black line). 


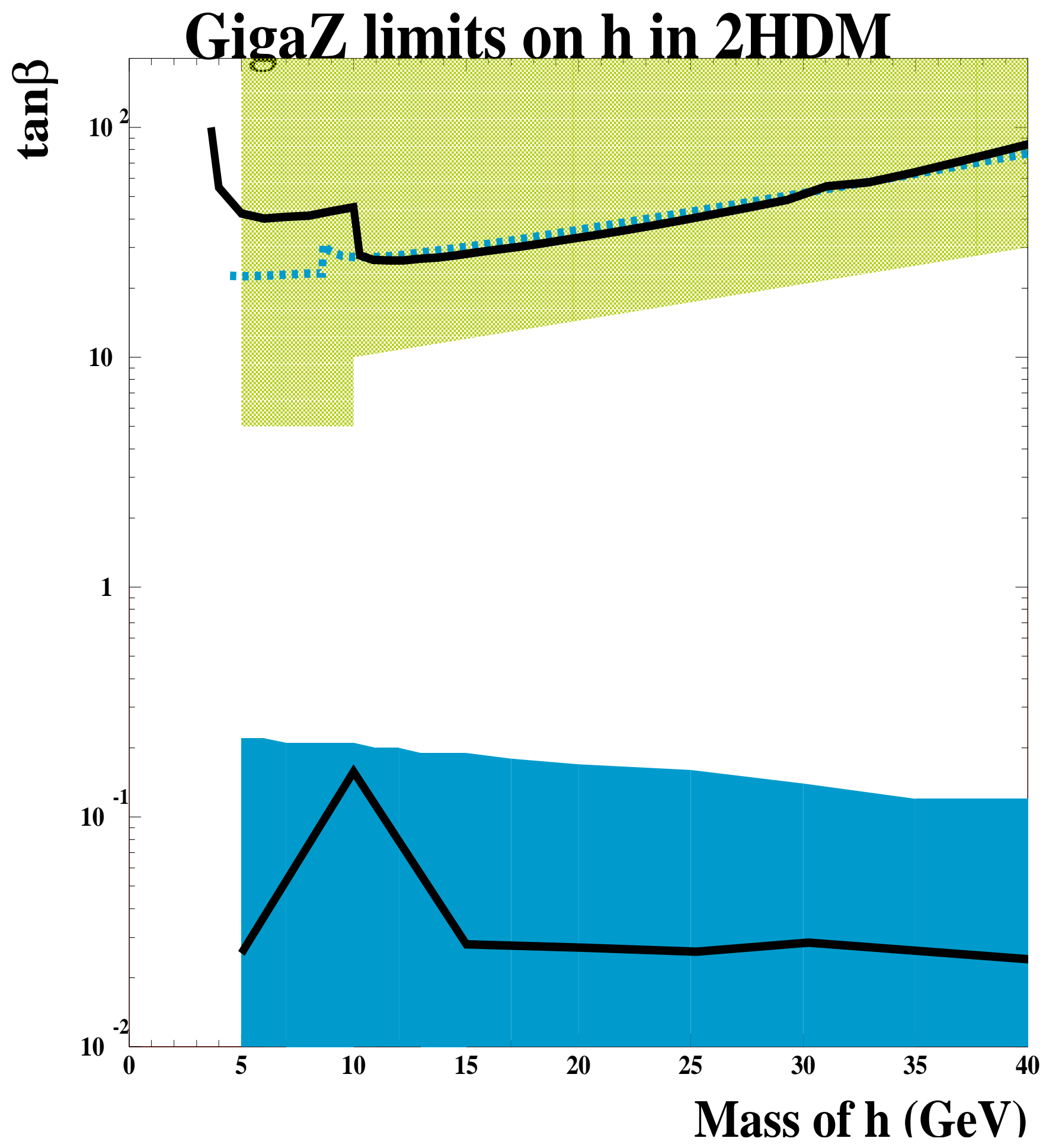

Figure 2: Potential exclusion range at GigaZ for the light scalar $h$ as a function of its mass and $\tan \beta$ (for $\sin (\beta-\alpha)=0$ ). The upper light (green) shaded region is excluded by the Yukawa process, the lower dark (blue) shaded region due to $Z \rightarrow h \gamma$. The upper limit from $Z \rightarrow h \gamma$ for $\tan \beta>1$ is indicated by the (blue) dotted line. Also shown is the current best limits obtained at LEP (black lines - upper from the Yukawa process and lower from the radiative $Z$ decay). 


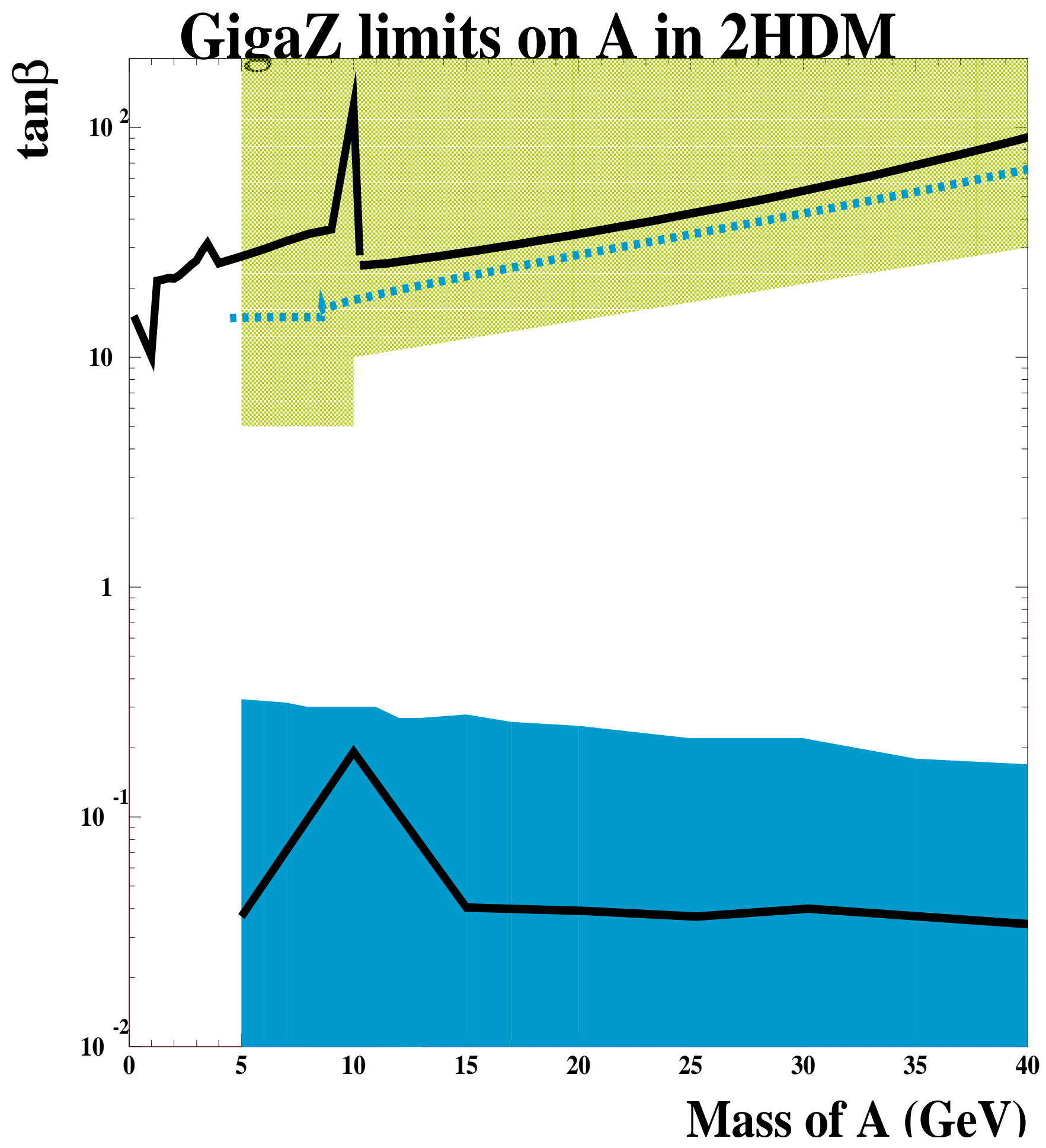

Figure 3: Potential exclusion range at GigaZ for the light scalar $A$ as a function of its mass and $\tan \beta$. The upper light (green) shaded region is excluded by the Yukawa process, the lower dark (blue) shaded region due to from $Z \rightarrow A \gamma$. The upper limit from $Z \rightarrow A \gamma$ for $\tan \beta>1$ is indicated by the (blue) dotted line. Also shown is the current best limits obtained at LEP (black lines- upper from the Yukawa proces and lower from the radiative $Z$ decay). 


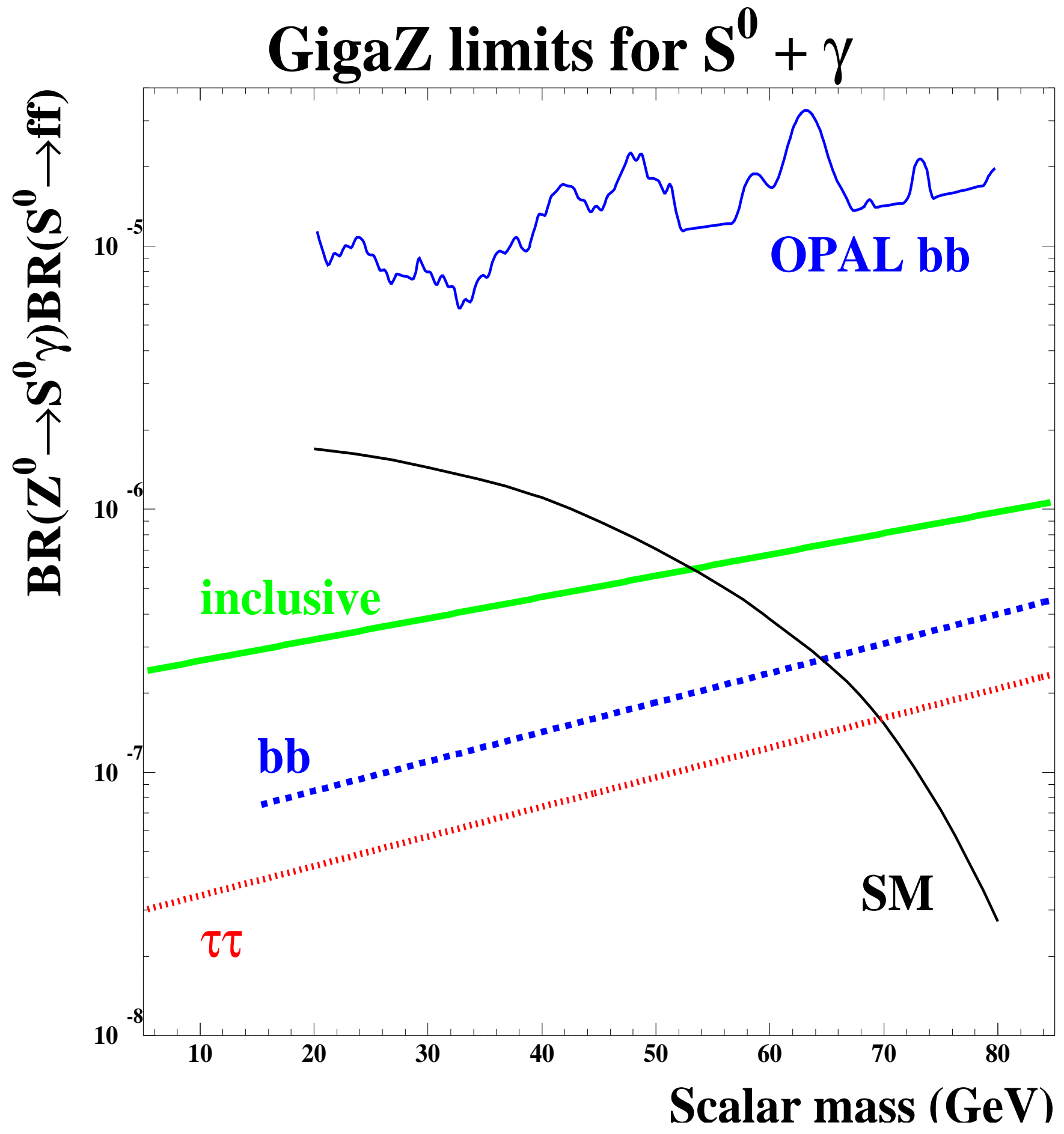

Figure 4: Potential sensitivity on the branching ratio $Z \rightarrow S+\gamma$ expected at GigaZ for various decay modes. The full (green) line shows the limits for decays into any kind of quarks or gluons (inclusive), the dashed (blue) into beauty quarks, and the dotted (red) into $\tau$ pairs. Also indicated are the current LEP reach for decays into bottom quarks [20] (narrow full/blue line) and the Standard Model prediction (black line). 